Results The frequency of APC, KRAS, NRAS, BRAF, CTNNB1 and RNF43 mutations were $76.4 \%, 61.8 \%, 9.1 \%, 3.6 \%$, $1.8 \%$ and $9.1 \%$. The most common RNF43 mutation was p. Gly659fs. RNF43 mutation was associated with MSI-H status $(p<0.001)$ and BRAF V600E mutation $(p<0.001)$. RNF43 and APC mutations were likely mutually exclusive. High pre-operative CEA level $\geq 10 \quad(p=0.013)$, high grade $(p=0.005)$, T4 tumours $(p=0.006)$, Stage IV cancer $(p<0.001)$ with distant metastasis $(p<0.001)$ were poor predictors of disease-free survival. High grade $(\mathrm{p}<0.001)$, T4 tumours $(\mathrm{p}<0.001)$, Stage IV cancers $(\mathrm{p}<0.001)$ with distant metastasis $(\mathrm{p}<0.001)$ were poor predictors of overall survival.

Conclusions The frequency of major mutations in Chinese population was stable. RNF43 mutation is likely anti-EGFR therapy resistant and is an alternative to targeted therapy. The current clinical classification of colorectal cancer and blood tests for disease monitoring were the most effective way to predict clinical prognosis. More research of larger sample size on the pathogenicity of RNF43 mutation and clinical correlation of major mutations is required.

\section{IDDF2018-ABS-0100 DECIPHERING MOLECULAR PROPERTIES OF HYPERMUTATED COLORECTAL AND GASTRIC CANCER}

Wangxiong Hu*. Cancer Institute (Key Laboratory of Cancer Prevention and Intervention, China National Ministry of Education), The Second Affiliated Hospital, Zhejiang University School of Medicine, China

\subsection{6/gutjnl-2018-IDDFabstracts. 10}

Background It is well-known that tumour is caused by somatic mutations. However, great mutational heterogeneity is observed both across cancer types (>1000 fold) and with a given cancer type, with a fraction of them harbour $>10$ mutations per $\mathrm{Mb}$, thus termed hypermutation. Hypermutated patients are suitable for PD-1 blockade with favourable prognosis. Nevertheless, other omics such as transcriptome and methylome in hypermutated samples remain poorly understood. Here, we try to determine the genome-wide effects of high mutation loads on transcriptome and methylome across two cancer types, namely colorectal cancer (CRC) and stomach adenocarcinoma (STAD).

Methods All tumour mRNA expression datasets (RNASeqV2) and DNA methylation data (HumanMethylation450) were obtained from The Cancer Genome Atlas. Known batch effects were corrected using the ComBat function implemented in the Bioconductor sva package. Differentially expressed gene (DEGs, false discovery rate (FDR) adjusted $P$-value $<0.05$ and fold change $>2$ ) analysis between hypermutated and nonhypermutated was performed by DEGSeq package for R/Bioconductor. Significantly differentially methylated site (DMS, FDR adjusted $P$-value $<1 \mathrm{E}-20$ and beta value change $>0.2$ ) was performed by limma package for R/Bioconductor.

Results Most hypermutated cases were driven by microsatellite instability-high (MSI-H). Meanwhile, 935 and 1,047 DEGs and 1604 and 53 DMSs were identified in CRC and STAD (hypermutated vs. non-hypermutated), respectively. A well-conceived five-gene signature (DNAI2, EPHA5, GAS2L1, RNH1, TAGLN3) was identified that could predict prognosis of STAD hypermutated patients with high performance (C-index: 0.84). Additionally, hierarchical clustering of expression and methylation profiles show that majority of CRC and STAD hypermutated samples were mixed and separated from their respective non-hypermutated samples, exceeding the boundary of tissue-specificity. Further in-detail exploration uncovered that the underlying molecular mechanism was related to perturbation of chromatin remodelling genes (ARID1A, NCOR1, and MLL1 $\sim 4$ ) both in CRC and STAD hypermutated samples.

Conclusions We thus concluded that MSI-H->chromatin remodelling genes inactivation- $>$ DNA methylation/expression variation axis shared in hypermutated samples was responsible for the consistent expression and methylation patterns across cancer types.

\section{IDDF2018-ABS-0104 MOLECULAR INSIGHTS INTO COLON MUCINOUS ADENOCARCINOMA}

Yanmei Yang*. Key Laboratory of Reproductive and Genetics, Ministry of Education, Women's Hospital, Zhejiang University, China

\subsection{6/gutjnl-2018-IDDFabstracts. 11}

Background Mucinous adenocarcinoma (MAC) is a histological subtype of colorectal cancer (CRC), in which account for 5\%$15 \%$ of all primary CRC. MAC is characterised by the formation of a tumour comprised of $>50 \%$ mucin. The prognostic value of MAC remains controversial; several studies report that MAC has a worse prognosis than nonmucinous adenocarcinoma (non-MAC), while others are not. Thus, it is important to decipher the molecular signature of MAC to better understand the unfamiliar subtype of the disease and improve the individualised management of patients with MAC.

Methods Here, we comprehensively characterised the somatic mutation, genome-wide transcriptional (mRNA and miRNA), and epigenetic (DNA methylation) profiles of the MAC, combined with correlative analyses of expression, methylation, and clinical data from the Cancer Genome Atlas. Differentially expressed gene (DEGs, false discovery rate (FDR) adjusted $P$ value $<0.05$ and fold change $>2$ ) analysis between MAC and non-MAC was performed by DEGSeq package for R/Bioconductor. Significantly differentially methylated site (DMS, FDR adjusted $P$-value $<1 \mathrm{E}-5$ and beta value change $>0.2$ ) was performed by limma package for R/Bioconductor.

Results Results showed that MAC harbours 3366 frequent mutational gene sets (FMGSs, apriori method), in sharp contrast to only 94 in non-MAC. Three-fold higher BRAF mutation rate in MAC than non-MAC was observed, and this may account for a worse prognosis in MAC. Notably, we found ATM had the same high mutation rate as TP53 in MAC, thus rescue of ATM was rather important because ATM acts upstream of TP53. In addition, hundreds of DEGs such as AQP3, MUC2, and FCGBP and 4 differentially expressed miRNAs were determined between MAC and non-MAC. Additionally, 1,926 DMSs that were associated with inflammatory response, cell adhesion, neutrophil chemotaxis, and immune response were identified as well.

Conclusions The huge molecular difference exists between $\mathrm{MAC}$ and non-MAC inspires us treating CRC in a more careful manner because we are driving toward a new era of precision medicine. In other words, the medical decision making used in MAC cannot simply copy from strategy adopted in non-MAC. 